\title{
Iniciativa emprendedora del recurso humano inactivo en el Establecimiento Penitenciario de Mediana Seguridad y Carcelario de Bucaramanga
}

Entrepreneurial initiative of the inactive human resource in the Penitentiary Establishment of Medium Security and Prison of Bucaramanga

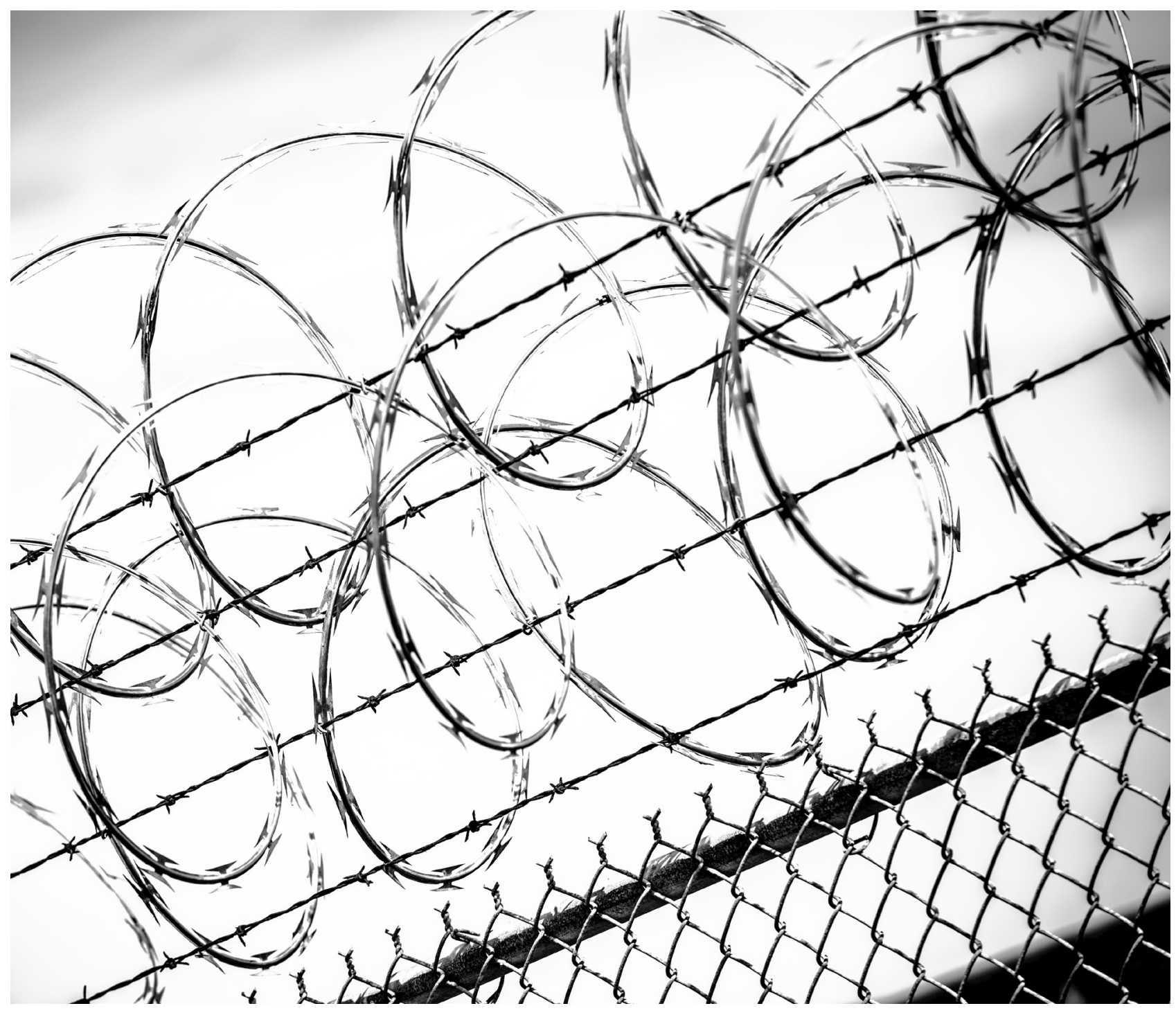




\title{
Iniciativa emprendedora del recurso humano inactivo en el Establecimiento Penitenciario de Mediana Seguridad y Carcelario de Bucaramanga ${ }^{1}$
} Entrepreneurial initiative of the inactive human resource in the Penitentiary Establishment of Medium Security and Prison of Bucaramanga

\author{
Youseline Garavito-Hernández², Jesús Alberto Córdoba-Campos ${ }^{3}$, \\ Reinaldo Ardila-Sequeda ${ }^{4}$
}

Artículo recibido en julio 18 de 2019; artículo aceptado en septiembre 24 de 2019

\begin{abstract}
Este artículo puede compartirse bajo la Licencia Creative Commons Atribución-NoComercial-Compartirlgual 4.0 Internacional y se referencia usando el siguiente formato: Garavito-Hernández, Y., Córdoba-Campos, J. \& Ardila-Sequeda, R. (2020). Iniciativa emprendedora del recurso humano inactivo en el Establecimiento Penitenciario de Mediana Seguridad y Carcelario de Bucaramanga. I+D Revista de Investigaciones, 15 (1), 57-66. DOI: https://doi.org/10.33304/revinv.v15n1-2020006
\end{abstract}

\section{Resumen}

El código penitenciario colombiano se encuentra regulado por la Ley 65 de 1993, que establece que las funciones y finalidades de la pena en Colombia son la protectora y la preventiva, pero su fin fundamental es la resocialización. Actualmente, se presenta en el país una crisis penitenciaria y carcelaria en el proceso de resocialización a la vida laboral del recluso, por su condición de expresidiario. En esta investigación se hace un análisis de las características personales y del entorno del recurso humano inactivo y su influencia en la iniciativa emprendedora en el Establecimiento Penitenciario de Bucaramanga, con el fin de proponer estrategias que favorezcan la resocialización de esta población vulnerable. El estudio tiene un enfoque cuantitativo no experimental, de corte transversal y de alcance relacional. Se comprobó que las características personales y del entorno global influyen positivamente en la iniciativa emprendedora del privado de la libertad, por lo que se propone la creación de una unidad de emprendimiento en el Establecimiento Penitenciario.

Palabras clave: Iniciativa emprendedora, recurso humano inactivo, recluso, establecimiento penitenciario.

\footnotetext{
'Artículo de investigación empírico, de enfoque cuantitativo, resultado de un proyecto de Maestría en Administración, perteneciente al área de ciencias sociales, desarrollado en los grupos de investigación PORTER y VIDOCQ, financiado por la Universidad de Investigación y Desarrollo (Bucaramanga, Colombia). Dirección: calle 9 n. ${ }^{\circ}$ 23-55, PBX: 6352525. Fecha de inicio: agosto de 2016. Fecha de terminación: diciembre de 2017.

2 Doctora en Administración y Dirección de Empresas. Investigadora Júnior del Grupo PORTER. Universidad de Investigación y Desarrollo (Bucaramanga, Colombia). Dirección: calle 9 n. 23-55, PBX: 6352525. ORCID ID: https://orcid.org/0000-0002-1644-9959. Correo electrónico institucional: ygaravito2@udi.edu.co.

${ }^{3}$ Magíster en Administración, profesional en Criminalística. Investigador del grupo VIDOCQ. Universidad de Investigación y Desarrollo (Bucaramanga, Colombia). Dirección: calle 9 n. 23-55, PBX: 6352525. ORCID ID: https://orcid.org/0000-0001-6822-4578. Correo electrónico institucional: jcordoba1@udi.edu.co.

${ }^{4}$ Magíster en Administración, abogado especializado en Derecho Penal. Investigador de la Universidad de Investigación y Desarrollo (Bucaramanga, Colombia). Dirección: calle 9 n. ${ }^{\circ}$ 23-55, PBX: 6352525. ORCID ID: https://orcid.org/0000-0001-8600-3234. Correo electrónico institucional: rardila1@ udi.edu.co.
} 


\begin{abstract}
The Colombian prison code is regulated by Law 65 of 1993, which establishes that the functions and purposes of punishment in Colombia are protective and preventive, but its fundamental purpose is the resocialization. Currently, there is a penitentiary and prison crisis in the process of resocialization to the working life of the prisoner, due to their condition as ex-convicts. This research is an analysis of inactive human resources, the personal characteristics, environment, and influence on the entrepreneurial initiative in the Penitentiary Establishment of Bucaramanga, in order to propose strategies that favor the resocialization of this vulnerable population. The study has a nonexperimental, quantitative, cross-sectional approach of relational scope. It was verified that the personal characteristics and the environment have a positive influence on the prisoner entrepreneur initiative, whereby the creation of an entrepreneurship unit in the Penitentiary Establishment is proposed.
\end{abstract}

Keywords: Entrepreneurial intention, inactive human resources, prisoner, penitentiary.

\section{Introducción}

El estudio del emprendimiento ha despertado en nuestra sociedad un gran interés en distintos grupos minoritarios, como lo son las mujeres cabeza de hogar, las personas de estratificación 1 y 2, los indígenas, los afrodescendientes y los individuos privados de la libertad, entre otros. Entre estos grupos, este estudio se enfoca en los individuos privados de la libertad, debido a que en el país se presenta una crisis en el proceso de reinserción laboral de esta población, lo que promueve la reincidencia delictiva y el aumento de la delincuencia en Colombia (Arias, 2019).

Es por ello que el Establecimiento Penitenciario y Carcelario de Bucaramanga, que se encuentra bajo la dirección y control del Instituto Nacional Penitenciario y Carcelario (INPEC), ubicado en el sector céntrico de la ciudad, se regula por un régimen progresivo que permite un seguimiento continuo del recluso. De esta forma, el régimen le permite brindar herramientas para alcanzar la resocialización del infractor de la ley penal, por medio de la disciplina, el trabajo, el estudio, la formación espiritual, la cultura, el deporte y la recreación, bajo un espíritu humano y solidario (Congreso de Colombia, 1993).

Así, formar empresas en el interior de los establecimientos carcelarios con aprovechamiento del recurso humano inactivo es una responsabilidad social ligada a los grandes fines del Estado (Torres, 2018). Para mejorar la situación descrita, se hizo necesario efectuar una investigación en la población reclusa del Establecimiento Penitenciario y Carcelario de Bucaramanga, a fin de identificar en ellos características particulares, en su entorno personal, familiar y global, para generar estrategias que permitan fomentar el emprendimiento.

No lejos de esta realidad, en los ámbitos nacional e internacional existen programas de formación que buscan explorar la capacidad emprendedora de las personas privadas de la libertad, y esto ha sido un esfuerzo de los entes gubernamentales y de la sociedad civil en general.

Por ejemplo, Magdalena (2013) señala que el personal privado de la libertad trabaja en los emprendimientos e incluso tiene motivaciones para la autogestión y realización de proyectos propios, no solo por el hecho de la rentabilidad económica, sino porque también el trabajo les permite "salir" de las condiciones de encierro en las que están normalmente, recreándose como sujetos de acción, capaces de imaginar. En esta lógica, el emprendimiento es una herramienta de construcción de nuevo espacio y tiempo, a la vez que una práctica económica y una posibilidad de resocialización.

En términos generales, hablar de iniciativa emprendedora implica aludir a un proceso dinámico en el que las personas identifican oportunidades para innovar y actúan transformando ideas en actividades prácticas dentro de un contexto social, cultural y económico en el que pueden desarrollar su espíritu emprendedor (Iruarrizaga \& Mendialdua, 2014; Nueno, 2011). Es así como en el contexto penitenciario es importante identificar aquellas personas privadas de la libertad que tengan ingenio o iniciativa que se evidencien en su cotidianidad penitenciaria, con el objeto de proporcionar ayuda y acompañamiento profesional que logren explorar oportunidades en este escenario.

Por tanto, se puede definir que en el mundo de los negocios el emprendedor es aquella persona que se caracteriza por ser un visionario creador que conduce un proceso de cambio, para lo cual descubre y crea nuevas oportunidades (Sastre, 2013). También se define como aquella persona que es innovadora, crea incertidumbre y perturba el equilibrio del mercado (Schumpeter, 1934), o como alguien que aprovecha las crisis empresariales y genera nuevas oportunidades, de modo que promueve la competitividad (Kirzner, 1973). En este orden de ideas, el rol del emprendedor es descubrir, evaluar y explotar las oportunidades que se presentan, ya sea creando nuevos productos o servicios, desarrollando nuevas estrategias, 
abriendo nuevos mercados o introduciendo nuevos procesos (Chirinos, Meriño, \& Martinez, 2018; Shane \& Venkataraman, 2000). Estudios realizados en los últimos años en relación con el emprendedor se han enfocado en el análisis de sus características personales y su entorno global y personal, así como en su influencia en la iniciativa emprendedora.

En relación con las características personales, se destacan factores psicológicos, como rasgos de la personalidad, capacidad para resolver problemas, eficiencia, características cognitivas como la intuición y capacidad de generalizar (Díez-Cuervo et al., 2005). Estas características cognitivas tienen que ver con la forma en que las personas explican los eventos o los resultados de los eventos, así como en la forma en que ellos se perciben en el entorno (Van Gelderen, Thurik \& Bosma, 2006). Estudios han encontrado que los emprendedores y los no emprendedores no tienen grandes diferencias en aspectos de personalidad; por este motivo, muchas investigaciones se han enfocado en el rol del proceso cognitivo en el emprendimiento (Baron, 1998).

Entre estas características, las más mencionadas son la necesidad de logro. McClelland (1961) fue el primero en afirmar que la necesidad de logro es la de mayor relevancia para el éxito empresarial, y Murray (1938) definió el término como aquello necesario para lograr algo difícil, lo que sirve para superar obstáculos y mejorar; también es necesaria para autorrealizarse y/o rivalizar y superar a otros. Otra dimensión de la personalidad que se examina en el contexto organizacional y en trabajos psicológicos es el locus de control. Estudios confirman que las personas con un fuerte locus de control interno se enfrentan mejor a las presiones en el trabajo, por lo cual están más satisfechas con sus actividades y pueden hacer frente a los cambios (Frank, Lueger \& Korunka, 2007). La tercera característica mencionada frecuentemente es la propensión al riesgo. Estudios empíricos han demostrado que los emprendedores se caracterizan en términos ideales por un nivel medio de propensión al riesgo (Pabón, 2011).

Así mismo, entre los rasgos personales del emprendedor también están los factores no psicológicos, como el conocimiento, la formación, las habilidades y la experiencia previa en la creación de empresas (Deakins \& Whittam, 2000). Otro factor no psicológico es el coste de oportunidad (pullvs. push). Se da un caso de push cuando, por ejemplo, faltan alternativas, como ser desempleado, ser despedido de una empresa o simplemente abandonar la empresa. Por tanto, la creación de una empresa es una oportunidad de empleo. $Y$ se dan casos de pull cuando se busca autonomía o desafío personal. También en la literatura se mencionan otras características no psicológicas, como que los padres sean o hayan sido emprendedores, el estado civil, la edad y el sexo, entre otros (Capelleras \& Genescá, 2003).
Además de las características personales, también está el entorno, que tiene una influencia en la decisión de un individuo de iniciar su carrera como emprendedor. Así, el entorno personal del emprendedor interviene en la iniciativa emprendedora, a través de factores como el alto estatus social, que facilita la decisión de desarrollar oportunidades de negocios, al permitir que el emprendedor sea más propenso en convencer a otros de los beneficios de sus proyectos, porque tendrá mejor acceso a recursos y puede sortear los riesgos con mayor facilidad. Asimismo, este autor menciona que la familia y la pertenencia a un grupo minoritario son otros factores de relevancia, al proveer al individuo de recursos complementarios, capacidades de gestión, redes de apoyo, fondos y garantías para desarrollar una gestión adecuada a sus intereses y los de su comunidad, ya que garantizan el éxito de su idea de negocio. Como idea complementaria, mantener estas redes de apoyo es importante para contribuir a la capacidad empresarial, a la decisión de crear una start-up, a la organización y adquisición de recursos. Además, al principio son genuinas, entrelazan intereses comerciales con compromisos sociales, pero con el tiempo varían, especialmente en las fases tempranas del desarrollo de la iniciativa de negocio (Jack, Drakopoulou \& Anderson, 2008).

En relación con el entorno global, la macroeconomía explica el desarrollo de las empresas, a través de variables como el crecimiento económico, la estabilidad económica, el tamaño del mercado, la legislación fiscal y las condiciones de la industria. En relación con esta última, incluye variables relevantes, como la necesidad de conocimiento; la intensidad en investigación y desarrollo; las condiciones de demanda; el ciclo de vida, años, consolidación y estructura de la industria; o la rentabilidad de las patentes. Estas pueden afectar la creación de nuevas empresas, como un resultado de la rentabilidad esperada. Se pueden afectar la intensidad de capital, la intensidad de la publicidad, la concentración y el tamaño mínimo de eficiencia de las empresas (Cuervo, 2005).

La financiación es otro factor del entorno que puede afectar la iniciativa emprendedora, debido a que está relacionado con el capital de la nueva compañía, que afecta de dos formas el desarrollo de la nueva empresa. Primero, a través del coste de capital que afecta a las decisiones de inversión, y segundo, por medio de las características de la financiación de las nuevas empresas. Asimismo, el Gobierno puede apoyar a los emprendedores promoviendo incentivos en cuanto a impuestos 0 subsidios, lo que reduce los problemas de incertidumbre en la explotación de las oportunidades empresariales. Por otra parte, el entorno geográfico es otro factor que influye en la iniciativa emprendedora, ya que la actividad empresarial puede estar organizada acorde con el grado de coordinación de diversas actividades, como la integración vertical de las empresas, las conglomeraciones y la cooperación entre las redes (Cuervo, 2005). 
En cuanto al entorno global, se encuentran características relacionadas con el entorno institucional, del que hacen parte factores que influyen en la protección de los derechos de propiedad de los inversores, regulaciones gubernamentales, inversión en $I+D$, inversión cultural y valores de la sociedad, como la legitimidad de la actividad emprendedora y la confianza. Otro importante factor del entorno institucional son las universidades que conceptualizan un sistema social de innovación y educación de cómo emprender, que no solo se puede considerar como un lugar de producción orientada al emprendimiento individual, sino también como un lugar de producción de mecanismos sociales que facilitan el nacimiento y crecimiento de negocios y empresas (Laukkanen, 2000). Entre otros factores del entorno estudiados en la literatura previa, están disponibilidad de capital de riesgo, presencia de empresarios con experiencia, mano de obra calificada, accesibilidad a proveedores, accesibilidad a clientes o nuevos mercados, influencias gubernamentales, proximidad a universidades, disponibilidad de tierras o instalaciones, acceso a transporte, actitud de la población de la zona, disponibilidad de servicios de apoyo y condiciones de vida (Bruno \& Tyebjee, 1982).

En este orden de ideas, teniendo en cuenta la problemática actual del Establecimiento Penitenciario de Mediana Seguridad y Carcelario de Bucaramanga y la importancia del emprendimiento para hacer frente a la situación carcelaria, en esta investigación se proponen la siguiente pregunta de investigación e hipótesis:

¿Cuáles son las características personales y del entorno que influyen en la iniciativa emprendedora del recurso humano inactivo del Establecimiento Penitenciario de Mediana Seguridad y Carcelario de Bucaramanga?

Hipótesis n. 1: Las características personales del recurso humano inactivo del Establecimiento Penitenciario de Mediana Seguridad y Carcelario de Bucaramanga influyen en su iniciativa emprendedora.

Hipótesis n. ${ }^{\circ}$ 2: Los factores del entorno personal del recurso humano inactivo del Establecimiento Penitenciario de Mediana Seguridad y Carcelario de Bucaramanga influyen en su iniciativa emprendedora.

Hipótesis n. ${ }^{\circ}$ 3: Los factores del entorno Global del recurso humano inactivo del Establecimiento Penitenciario de Mediana Seguridad y Carcelario de Bucaramanga influyen en su iniciativa emprendedora.

Para dar respuesta a la pregunta de investigación y contrastar las hipótesis, se realizó un análisis descriptivo, correlacional y de regresión logística. A continuación, se presentan las principales variables de estudio con sus respectivas definiciones:
Variable dependiente

Iniciativa emprendedora: Es un proceso social y dinámico en el que los individuos, solos o en colaboración, identifican oportunidades para innovar y actúan transformando las ideas en actividades prácticas dentro de un contexto social, cultural o económico (Nueno, 2011).

\section{Variables independientes}

Características personales del emprendedor: Son cualidades esenciales que conforman los atributos básicos del talento humano, tales como la capacidad para resolver problemas, cognición, necesidad de logro, eficiencia, locus de control interno, conocimiento, formación, habilidades, experiencia, coste de oportunidad, edad y estado civil (Franco \& Prata, 2019; Kurniawan, Basri, Yudoko, \& Umbara, 2019; Sastre, 2013).

Las características del entorno personal del emprendedor: El entorno del emprendedor es considerado como una de las condiciones del contexto en el que se desenvuelve el emprendedor. Cuenta con características particulares y relevantes, como el estatus social, las relaciones sociales en la familia y las redes sociales (Franco \& Prata, 2019; Kurniawan et al., 2019; Sastre, 2013).

Características del entorno global del emprendedor: El entorno global permite efectuar comparaciones del emprendedor frente al contexto global $y$ macroeconómico, tales como apoyo financiero, políticas sociales y programas gubernamentales en educación y formación, infraestructura, normas sociales y culturales (Franco \& Prata, 2019; Kurniawan et al., 2019; Sastre, 2013).

\section{Metodología}

\section{Tipo de estudio}

El estudio se estructuró bajo un enfoque cuantitativo. Se realizó la recolección datos y su análisis partiendo de variables como la iniciativa emprendedora, los factores individuales y del entorno del emprendedor, con el fin de llegar a conclusiones aplicables a toda una población, a partir de la selección de una muestra representativa. Por otro lado, el estudio tuvo un diseño no experimental, dado que no realizó manipulación de las variables independientes. Asimismo, fue transversal o transaccional, puesto que esta investigación se practicó mediante la aplicación de un cuestionario una sola vez, con el fin de reunir datos sobre las variables que fueron objeto de análisis. Por último, este estudio es de tipo explicativo, ya que su interés se centra en explicar el efecto de los factores individuales y del entorno del emprendedor que influyen en la iniciativa emprendedora del recurso humano inactivo del centro penitenciario de Bucaramanga (Hernández, Fernández, \& Baptista, 2006). 


\section{Participantes}

Esta investigación contó con la participación de los internos recluidos en el Establecimiento Penitenciario de Mediana Seguridad y Carcelario de Bucaramanga. La aplicación del instrumento se realizó a la población ubicada en tres sectores: los internos que se encuentran en los pabellones de seguridad, en las áreas de talleres $y$ en el sector del área educativa. Se escogieron estos sectores debido a la facilidad de cooperación de los reclusos en estas actividades y por estar en clasificación de mediana seguridad. A la fecha, la población de presos está conformada por 2800 personas. Por ser un estudio de caso, en cada pabellón se entrevistó a 30 reclusos, para un total de 90 internos encuestados. Esto con el fin de reunir el tamaño mínimo que se requiere para una investigación de tipo correlacional, debido a que un grupo menor a esta cifra podría brindar un estimado inexacto de relación existente entre las variables. La técnica de muestreo fue la de aleatorio simple, que consiste en un procedimiento de muestreo probabilístico que da a cada persona de la población objeto de estudio la misma probabilidad de ser seleccionada (Hernández et al., 2006).

\section{Materiales e instrumentos}

El instrumento que se utilizó para la recolección de información fue una encuesta, elaborada con dieciocho preguntas tendientes a evaluar la iniciativa emprendedora, las características personales de la población reclusa y el entorno personal y global de esta. El instrumento construido se basó en estudios previos de iniciativa emprendedora, especialmente del Global Entrepreneurship Monitor, que anualmente mide la iniciativa emprendedora en el mundo; y se validó a través del método Delphi.

\section{Observación natural}

Para el trabajo de campo se consultaron todos los lineamientos y protocolos de seguridad adoptados por el Establecimiento Penitenciario. Asimismo, la investigación contó con el visto bueno y aval del entonces director del Establecimiento Penitenciario, el capitán Julio Enrique Pardo Fandiño. Para eso, fue necesario generar previamente las solicitudes correspondientes y de esta forma lograr ingresar a la parte interna del centro penitenciario, el día 11 de mayo de 2018. En tal jornada, se elaboró inicialmente una prueba piloto en la biblioteca de la comunidad Nuevos Horizontes con cuatro internos. Posteriormente se aplicó el instrumento al total de la muestra.

\section{Procedimiento}

Para analizar las características personales y del entorno y contrastar las hipótesis, fue necesario realizar algunas actividades, a través de los siguientes objetivos específicos de investigación.
Objetivo específico $n .^{\circ}$ 1: Realizar un estudio descriptivo de las características personales y del entorno del recurso humano inactivo del establecimiento carcelario de Bucaramanga.

Actividades:

a) Recoger una muestra representativa de datos a través de la encuesta.

b) Diagnosticar la situación actual de los reclusos en cuanto a su iniciativa emprendedora.

Objetivo específico $n .^{\circ}$ 2: Analizar la influencia de las características personales y del entorno del recurso humano inactivo en la iniciativa emprendedora, a través de un análisis correlacional y explicativo.

Actividades:

a) Verificar la fiabilidad de los datos.

b) Realizar un análisis de regresión logística para contrastar las hipótesis.

c) Realizar el análisis correlacional con el objeto de conocer la relación entre las variables de estudio.

\section{Resultados}

Del diagnóstico obtenido, en relación con las características personales de los individuos privados de la libertad recluidos en el Establecimiento Carcelario de Bucaramanga, se evidenció que el $56 \%$ de los encuestados cuenta con estudios secundarios, frente a un $24 \%$ con estudios de primaria y un $20 \%$ con estudios superiores. Asimismo, el 53,33\% de los encuestados considera la experiencia como un factor determinante a la hora de emprender y no perciben la falta de educación como un factor que pueda impedir iniciar un emprendimiento.

Por otro lado, el $91,11 \%$ considera tener ideas para emprender, lo que es un factor que favorece el inicio del proceso emprendedor. Además, el 52,2\% es consciente de que se deben asumir riesgos al momento de emprender y que la toma de riesgos es una actitud básica para emprendedores. Por ello, un alto porcentaje $(88,88 \%)$ considera tener confianza en sí mismo, aunque haya riesgos u oportunidades, lo que es un factor clave para pasar de la idea a la acción. En conclusión, la mayoría $(91,11 \%)$ considera importante el emprendimiento frente a la opción de trabajar como empleado, lo que comprueba que en esta población se cumple la tendencia mundial del autoempleo para la población con menos oportunidades.

En relación con el diagnóstico de las características del entorno, el $75,5 \%$ conoce en su familia personas emprendedoras, de modo que es un factor que aporta al 
emprendimiento. Por otro lado, el $43,3 \%$ considera que no es necesario asociarse con personas o empresas para emprender un negocio; están en contra de la filosofía asociativa, que pretende generar mayores beneficios con la unión de pequeños esfuerzos. Asimismo, un $65,5 \%$ tiene la percepción de que no necesita de un estatus social a la hora de emprender un negocio.

Por otro lado, el $45,55 \%$ considera importante el factor dinero a la hora de emprender e identifica como principal fuente de financiación la familia y amigos. A pesar de ello, un $52,2 \%$ es consciente de la necesidad de utilizar los servicios financieros para emprender en Colombia. Por tanto, el 73,33\% identifica la red de apoyo con familiares y amigos de relevancia, ya que la mayoría $(86,67 \%)$ percibe que ellos pueden oírlos, aportarles nuevas ideas y proporcionarles recursos económicos.

Finalmente, el $53,3 \%$ de los reclusos señala que en el penal puede expresar sus ideas de emprendimiento; por tanto, es necesario fortalecer este aspecto para la creación de iniciativas de negocio. Además, el $73,3 \%$ reconoce que estar privado de la libertad es una oportunidad para desarrollar sus ideas de emprendimiento, de modo que es un factor que favorece la iniciativa empresarial dentro del Establecimiento Penitenciario. Asimismo, el 64,4\% tiene conocimiento de la existencia de programas de emprendimiento dentro del establecimiento carcelario, y la mayoría (98\%) se encuentra realizando alguna actividad de redención en el penal.

\section{Análisis de fiabilidad}

El análisis de fiabilidad permite conocer la confiabilidad del instrumento de medición utilizado para tener certeza de los datos recolectados. El método más utilizado es el Alfa de Cronbach. Este oscila entre 0 y 1 . El valor mínimo aceptable para el coeficiente Alfa de Cronbach es 0,7 (Molina, Aranda, Flores \& López, 2013). Para este estudio, el Alfa de Cronbach fue de 0,783; por tanto, el instrumento es confiable.

\section{Análisis de regresión}

Para el análisis de regresión se tuvo en cuenta el valor obtenido para las variables dependientes e independientes, que permitieron comprobar las hipótesis planteadas sobre la relación de la iniciativa emprendedora con las características personales, del entorno personal y global del emprendedor. El modelo de regresión se presenta en la Figura 1; y en la Tabla 1, los resultados de la regresión.

Del anterior modelo de regresión es posible concluir que la intención emprendedora está influenciada en mayor medida por las características personales y el entorno global del recurso humano inactivo del Establecimiento
Penitenciario. Por otro lado, se presenta una influencia negativa y no significativa del entorno personal. Esto puede deberse al poco contacto que mantiene el recluso con su entorno personal al estar recluido en la cárcel. Por tanto, se contrastaron de forma positiva y significativa las hipótesis 1 y 3 , mientras que no se pudo confirmar la hipótesis 2 , al no ser significativa.

$$
Y=0.574 X 1-0.211 X 2+0.195 \times 3+0.041
$$

Donde,

$Y=$ intención emprendedora

$X 1=$ características personales

$X 2=$ Entorno Personal

X3 = Entorno Global

Figura 1. Modelo de regresión. Fuente: Autores.

Tabla 1

Análisis de regresión

\begin{tabular}{lccccc}
\hline \multirow{2}{*}{ Modelo 1 } & \multicolumn{2}{c}{$\begin{array}{c}\text { Coeficientes no } \\
\text { estandarizados }\end{array}$} & Coef B & t & Sig. \\
\cline { 2 - 3 } & $\mathbf{B}$ & $\begin{array}{c}\text { Error } \\
\text { Estándar }\end{array}$ & & & \\
\hline $\begin{array}{l}\text { Constante } \\
\text { Escala }\end{array}$ & 0.041 & 0.546 & & 0.076 & 0.940 \\
$\begin{array}{l}\text { características } \\
\text { personales }\end{array}$ & 0.574 & 0.154 & 0.397 & 3.726 & 0.000 \\
$\begin{array}{l}\text { Escala } \\
\text { Entorno }\end{array}$ & -0.211 & 0.114 & -0.216 & -1.858 & 0.067 \\
$\begin{array}{l}\text { Personal } \\
\text { Escala } \\
\text { Entorno }\end{array}$ & 0.195 & 0.094 & 0.232 & 2.062 & 0.042 \\
Global & & & & & \\
\hline
\end{tabular}

Nota: *Variable dependiente: Escala intención emprendedora. Fuente: Autores.

\section{Análisis de correlación}

En este análisis se procedió a identificar las correlaciones de variables más destacadas. Para ello, es necesario aclarar que la correlación de las variables descritas en el presente estudio arrojó 406 combinaciones, cuyos principales resultados se presentan a continuación. Es necesario aclarar que para su desarrollo se utilizó el coeficiente de correlación de Pearson, en el que las correlaciones más cercanas a 1 serán más fuertes.

En la Tabla 2, para el caso de las características personales, la variable dependiente Motivación intrínseca presenta una correlación positiva media (coeficiente Pearson entre 0,25 y 0,50), con la formación académica y la propensión a asumir riesgos. Mientras que, por otro lado, presenta una correlación positiva muy débil con la experiencia al emprender (coeficiente Pearson entre 0,10 y 0,24). Por otra parte, existe una correlación negativa considerable entre la variable dependiente perspectiva de emprender y la capacidad de escuchar a otros, lo que indica que 
las personas consideran importante escuchar, pero de igual forma no tienen pensado realizar sus iniciativas de emprendimiento (ver Tabla 2).

Tabla 2

Análisis correlación características personales

\begin{tabular}{ccccc}
\hline \multicolumn{5}{c}{ Características personales de la población reclusa } \\
\hline & $\mathrm{I}$ & $\mathrm{II}$ & $\mathrm{III}$ & $\mathrm{IV}$ \\
& & & \\
Motivación intrínseca & $.225^{*}$ & $.270^{*}$ & .121 & \\
$\begin{array}{c}\text { Perspectiva de } \\
\text { emprendimiento }\end{array}$ & & & & $-.337^{* *}$ \\
& & & &
\end{tabular}

Nota: ${ }^{*} \mathrm{p}<0.05,{ }^{* *} \mathrm{p}<0.01$. La abreviatura I se refiere a "Formación académica"; la II, a "Propensión asumir riesgo"; la III, a "experiencia previa en creación de empresas, y la IV, a "capacidad de escuchar a otros". Fuente: Autores.

En la Tabla 3 se identifica la correlación positiva débil (coeficiente Pearson entre 0,10 y 0,24 ) entre la variable dependiente emprendimientos previos y la variable independiente libertad de expresión en el penal, lo que indica que los emprendedores sienten que sus ideas son atendidas. Se muestra también la correlación negativa débil entre el emprendimiento posterior a la salida de la cárcel y la existencia de emprendedores en la familia. Finalmente, se observa una correlación negativa débil entre el emprendimiento durante la estancia en la cárcel y la percepción del estatus social para emprender, lo que indica que las personas, en la medida que tienen una idea, sienten que no necesariamente deben pertenecer a un estatus social definido (ver Tabla 3).

Tabla 3

Análisis correlación características del entorno personal

\begin{tabular}{cccc}
\hline \multicolumn{4}{c}{ Características del entorno personal } \\
\hline Emprendimientos & $\mathrm{I}$ & $\mathrm{II}$ & $\mathrm{III}$ \\
previos & & & $.224^{*}$ \\
Perspectiva de & $-.272^{* *}$ & & \\
emprendimiento & & & \\
$\begin{array}{c}\text { Emprendimiento en } \\
\text { el penal }\end{array}$ & & $-.262^{*}$ & \\
\hline
\end{tabular}

Nota: ${ }^{*} \mathrm{p}<0.05,{ }^{* *} \mathrm{p}<0.01$. La abreviatura I se refiere a "Emprendedores en la familia"; la II, a "Estatus social en el emprendimiento", y la III, a "Libertad de expresión en el penal". Fuente: Autores.

Finalmente, en la Tabla 4 la variable dependiente motivación intrínseca tiene una correlación positiva débil con la variable necesidad de recursos económicos para emprender, mientras que guarda una correlación positiva media con las variables realización de actividad de redención en el penal y experiencia al emprender. Por otra parte, la mayor relación de variables de forma indirecta está entre las variables realización de actividad de redención de pena y motivación intrínseca; es decir, los encuestados que realizan actividades de redención no lo hacen por iniciativa propia, sino por la dinámica del sistema, y aquellos que no realizan actividades consideran que se requiere un alto nivel de iniciativa para desarrollar sus ideas de emprendimiento (ver tabla 4).

Tabla 4

Características del entorno global

\begin{tabular}{lccccc}
\hline \multicolumn{5}{c}{ Características del entorno global } \\
\hline & $\mathrm{I}$ & $\mathrm{II}$ & $\mathrm{III}$ & $\mathrm{IV}$ & $\mathrm{V}$ \\
$\begin{array}{l}\text { Motivación } \\
\text { Intrínseca }\end{array}$ & & $.272^{* *}$ & $-.518^{* *}$ & & $-.361^{* *}$ \\
$\begin{array}{l}\text { Experiencia } \\
\text { al emprender }\end{array}$ & $.220^{*}$ & & $.293^{* *}$ & $-.361^{* *}$ & \\
$\begin{array}{l}\text { Perspectiva } \\
\text { de }\end{array}$ & & $-.268^{*}$ & & & \\
emprender & & & & & \\
\end{tabular}

Nota: ${ }^{*} p<0.05,{ }^{* *} p<0.01$. La abreviatura I se refiere a "Servicios del sistema financiero colombiano"; la II, a "Necesidad de recursos económicos para emprender"; la III, a "Actividades de redención de pena en el penal"; la IV, a "Iniciativa para dar comienzo actividades de redención de pena en el penal", y la V, a "alguna vez ha emprendido". Fuente: Autores.

\section{Conclusiones}

En Colombia se presenta una crisis penitenciaria y carcelaria en el proceso de resocialización a la vida laboral del recluso, por su condición de expresidiario. En esta investigación se hace un análisis de las características personales y del entorno del recurso humano inactivo y su influencia en la iniciativa emprendedora en el Establecimiento Penitenciario de Bucaramanga, con el objeto de proponer estrategias que permitan la resocialización de esta población minoritaria.

Con los hallazgos del análisis estadístico se concluyó que las variables formación académica y la capacidad de escuchar deben ser fortalecidas en el interior del centro penitenciario. Para ello, se puede contar con el apoyo del Instituto Educativo San Juan Bosco, que cuenta con la autorización del Ministerio de Educación Nacional para desarrollar este tipo de capacitaciones a esta población. Además, el estudio arrojó una influencia positiva de las características del entorno global con la iniciativa emprendedora del recluso, lo que hace necesario motivar a la empresa privada y a entidades públicas para desarrollar convenios que incidan positivamente en la obtención de recursos económicos para el apoyo de las ideas y programas de emprendimiento en el Establecimiento Penitenciario. Por otro lado, en el análisis de regresión lineal se puede apreciar que las características del entorno personal no influyen de forma positiva y significativa en la iniciativa emprendedora, 
debido al poco contacto que tienen los presos con sus grupos de interés, como lo son familiares y amigos.

Así mismo, se destaca la necesidad de fortalecer los aspectos de mentalidad y cultura emprendedora. Para ello, es necesario el desarrollo de asesorías especializadas, búsqueda de financiación y fortalecimiento de las redes de apoyo, que resultan ser de importancia para los internos, pues, antes que emprendedores o reclusos, son personas o seres sociales con serias dificultades que los llevaron al contexto carcelario. Así, como propuesta de mejora se contempla la creación de una unidad de emprendimiento especializada, a través de la vinculación de las universidades de la ciudad que tengan algún tipo de convenio con el Instituto Nacional Penitenciario y Carcelario (INPEC).

Estos resultados están acordes con estudios previos en los que se sustenta que la educación genera efectos positivos para los emprendedores, porque permiten que los avances y los nuevos conocimientos sean conocidos por estos. De esta manera, se favorece la transformación de ideas en actividades comerciales; además, la formación académica favorece la reducción de las desigualdades, lo que crea un entorno más propicio para el emprendedor (Keeble, Bryson, \& Wood, 1993; Lucas, 1988). En referencia al entorno del emprendedor, Stevenson (2000) sostiene que este desempeña un papel crucial, y que es más probable que un individuo comience a tener actitudes emprendedoras si actúa en un contexto que favorece el reconocimiento de la oportunidad.

Por lo anterior, la tendencia en el ámbito mundial es la de que los Establecimientos Penitenciarios desarrollen programas educativos en emprendimiento que preparen a los reclusos para la reintegración en trabajo y sociedad. Es así que la educación empresarial en prisión y las redes de apoyo podrían ser eficaces para transformar las actitudes de los presos hacia ellos mismos y hacia su entorno, lo que les permitirá creer en sus capacidades para el desarrollo de ideas de negocios, y con esto se reducirían los índices de reincidencia delictiva y de delincuencia en Colombia.

Como futuras líneas de investigación se propone desarrollar este análisis en otras poblaciones minoritarias, como lo son las mujeres cabeza de hogar, personas de estratificación 1 y 2 , indígenas y afrodescendientes, entre otros. Asimismo, se plantea replicar este estudio en los Establecimientos Penitenciarios Zona Oriente, de modo que se obtengan datos más robustos para proponer estrategias que puedan generalizarse para esta población minoritaria. En caso de gestionar estos tipos de estudios, se sugiere, tal como se realizó en el presente estudio, contrastar los contenidos teóricos con el contexto, para la creación de instrumentos acordes a las necesidades del objeto de estudio.
La principal limitación del trabajo fue la imposibilidad de realizar una muestra realmente aleatoria, ya que, por las condiciones de seguridad, fue posible solo la aplicación del instrumento en aquellos internos que hacen parte del proceso de tratamiento penitenciario. El acceso a los demás es complejo, y esto limita la recolección de información.

\section{Agradecimientos}

Los más sinceros agradecimientos a las instituciones que participaron en el desarrollo de este proyecto, en especial al Instituto Nacional Penitenciario y Carcelario (INPEC), por facilitar el acceso para la investigación.

\section{Referencias}

Arias, G. E. (2019). Políticas de resocialización en el sistema carcelario en Colombia en el periodo 2015 al 2017. Recuperado de https://repository.ucatolica.edu.co/ handle/10983/23249

Baron, R. A. (1998). Cognitive mechanisms in entrepreneurship: why and when entrepreneurs think differently than other people. Journal of Business Venturing, 13(4), 275-294. https://doi. org/10.1016/S0883-9026(97)00031-1

Bruno, A. V., \& Tyebjee, T. T. (1982). The environment for entrepreneurship. In Encyclopedia of entrepreneurship 2 (Vol. 2, pp. 288-315). C. A. Kent, D. L. Sexton, \& K. H. Vesper (Eds.). Englewood Cliffs, NJ: Prentice-Hell.

Capelleras, J., \& Genescá, E. (2003). Formación y empleo por cuenta propia: Un análisis comparativo a nivel internacional. Creación de empresas: homenaje al profesor José María Veciana Vergés. Servei de Publicacions.

Chirinos, Y., Meriño, V., \& Martinez, C. (2018). Emprendimiento sostenible: una opción para el crecimiento local. I+ D Revista de Investigaciones, 11(1), 116-128. https://doi.org/10.33304/revinv. v11n1-2018009

Congreso de Colombia. Código Penitenciario y Carcelario. Ley 65 de 1993, artículo 10 (1993).

Cuervo, A. (2005). Individual environmental determinants of entrepreneurship. International Entrepreneurship and Management Journal, 1(3), 293-311.

Deakins, D., \&Whittam, G. (2000). Business start-up: theory, practice and policy. Enterprise and Small Business Principles, Practice and Policy, 115-131.

Díez-Cuervo, A., Muñoz-Yunta, J. A., Fuentes-Biggi, J., Canal-Bedia, R., Idiazábal-Aletxa, M. A., Ferrari-Arroyo, M. J., ... \& Artigas-Pallarés, J. (2005). Guía de buena práctica para el diagnóstico de los trastornos del espectro autista. Revista de Neurología, 41(5), 299-310.

Franco, M., \& Prata, M. (2019). Influence of the individual characteristics and personality traits of the founder on the performance of family SMEs. European Journal of 
International Management, 13(1), 41-68.

Frank, H., Lueger, M., \& Korunka, C. (2007). The significance of personality in business start-up intentions, start-up realization and business success. Entrepreneurship \& Regional Development, 19(3), 227-251.

Hernández, R., Fernández, C., \& Baptista, P. (2006). Metodología de la investigación (Vol. 4). México.

Iruarrizaga, J. H., \& Mendialdua, A. B. (2014). Financiación del proceso emprendedor. Ediciones Pirámide.

Jack, S., Drakopoulou, S., \& Anderson, A. (2008). Cange and the development of entrepreneurial networks over time: a processual perspective. Entrepreneurship \& Regional Development, 20(2), 125-159. https://doi. org/10.1080/08985620701645027

Keeble, D., Bryson, J., \&Wood, P. (1993). Business networks, small firm flexibility and regional development in UK business services. Entrepreneurship \& Regional Development, 5(3), 265-278. https://doi. org/10.1080/08985629300000016

Kirzner, I. M. (1973). Competition and Entrepreneurship. University of Chicago Press.

Kurniawan, M. E. H., Basri, M. H., Yudoko, G., \& Umbara, A. N. (2019). Do entrepreneurship students have an intention to become an entrepreneur? Journal of Entrepreneurship Education, 22(2), 1-14.

Laukkanen, M. (2000). Exploring alternative approaches in high-level entrepreneurship education: creating micro-mechanisms for endogenous regional growh. Entrepreneurship \& Regional Development, 12(1), 2547. https://doi.org/10.1080/089856200283072

Lucas, R. (1988). On the mechanics of economic development. Journal of monetary economics. Journal of Monetary Economics, 22(1), 3-42. https:// doi.org/10.1016/0304-3932(88)90168-7

Magdalena, J. (2013). Emprendimientos productivos en cárceles. La construcción de valor más allá de lo económico. In VII Jornadas Santiago Wallace de Investigación en Antropología Social. Sección de Antropología Social. Instituto de Ciencias Antropológicas. Facultad de Filosofía y Letras, UBA. Recuperado de https://www.aacademica.org/000-063/244

McClelland, D. C. (1961). Achieving society (No. 15). Simon and Schuster.

Molina, J. A. B., Aranda, L. L., Flores, M. E. H., \& López, E. J. (2013). Utilización del alfa de Cronbach para validar la confiabilidad de un instrumento de medición de satisfacción del estudiante en el uso del software Minitab MISP. In In 11th LACCEI Latin American and Caribbean Conference for Engineering and Technology (LACCEI'2013) "Innovation in Engineering, Technology and Education for Competitiveness and Prosperity" (pp. 14-16).

Murray, H. A. (1938). Explorations in personality: a clinical and experimental study of fifty men of college age. Oxford, England: Oxford Univ. Press.

Nueno, P. (2011). Iniciativa emprendedora y empresa familiar: emprendiendo a través de las generaciones.
Universia Business Review, (32), 96-101.

Pabón, R. M. (2011). Estudio de actitudes emprendedoras con profesionales que crearon empresa. Revista Escuela de Administración de Negocios, (71), 70-83.

Sastre, R. F. (2013). La motivación emprendedora y los factores que contribuyen con el éxito del emprendimiento. Ciencias Administrativas, (1), 1-10.

Schumpeter, J. (1934). The Theory of Economic Development. Harvard University Press, Cambridge, MA.

Shane, S., \& Venkataraman, S. (2000). The promise of entrepreneurship as a field of research. Academy of Management Review, 25(1), 217-226. https://doi. org/10.5465/amr.2000.2791611

Stevenson, H. (2000). Why the entrepreneurship has won! In Coleman White Paper USASBE Plenary Address (pp. 1-8). Recuperado de http://scholar.google.com/scho $\operatorname{lar} ? \mathrm{hl}=\mathrm{en} \& b \operatorname{tnG}=$ Search \&q=ititle:WHY+ETREPRENEU RSHIP+HAS+WON\#0

Torres, J. A. (2018). Incursión de la empresa privada como alternativa para la motivación laboral en el Establecimiento Penal de Lurigancho 2018. Universidad César Vallejo. Recuperado de http://repositorio.ucv. edu.pe/handle/UCV/19644

Van Gelderen, M., Thurik, R., \& Bosma, N. (2006). Success and risk factors in the pre-start up pahse. Small Business Economics, 24(4), 365-380. 